\title{
MS01-P04 | METALJET SOURCE ENABLING ADVANCED PROTEIN CRYSTALLOGRAPHY
}

Espes, Emil (Excillum AB, KISTA, SWE); Hållstedt, Julius (Excillum AB, Kista, SWE)

X-ray diffraction data from protein and bio crystals are typically of a much lower resolution and quality compared to measurements in chemical crystallography. This is mainly due to large molecules, poor crystalline quality in combination with very light atoms embedded in large amount of water. At the same time, unit cell size is extremely large and thus the Bragg reflections are very closely packed together. Protein crystallographers must rely on the strongest $X$-ray sources to combat the issues of air sensitivity, small crystals, weakly diffracting and densely packed reflections. Traditionally, a high brilliance synchrotron has been used to measure full protein data leading for structure determination, whilst home laboratory instruments have been used only for protein screening to identify the preferred crystals for measurement at the synchrotron. High brightness X-ray compact sources, such as the MetalJet have not only improved crystal screening but also made it possible to obtain publishable data from extremely difficult experiments such as GPCR membrane proteins. In this communication we will show a number of different examples how the MetalJet X-ray source can be a game changer for home laboratory macro molecular diffraction. 